\title{
The Author's Reply: Determinants of Functional Outcomes Using Clinical Pathways for Rehabilitation After Hip Fracture Surgery
}

As the older population increases in number, physical impairments and disability associated with falls and fragility fractures constitute major threats to health and increase healthcare and socioeconomic burdens. Therefore, we need to be aware of the global trends in fragility fractures and their impact on health care for older adults. In recent years, many academic societies have continued to promote appropriate postfracture rehabilitation and to emphasize the prevention of falls and repeated fractures. In fact, the rehabilitation of acute hip fractures in Korea has not been well established due to limited integration of care among the orthopedic, geriatric, and rehabilitation specialties. Despite the difficult circumstances, we developed clinical pathways for rehabilitation of patients with acute hip fractures; furthermore, we accumulated the data of patients who showed significant functional improvement following rehabilitation management in these populations. ${ }^{1)}$ One of the reasons why the results of our study were reported in Annals of Geriatric Medicine and Research was to share our experience with other researchers.

The points of view raised by Prof. Kim are appropriate with regards to study limitations, for example, the possibility of selection bias. ${ }^{2)}$ Because this study was a retrospective cohort study based on the medical records of one hospital, considerable follow-up losses were unavoidable. We developed a critical pathway for hip fracture rehabilitation and since 2007, have introduced it into clinical practice as a collaborative model with orthopedic surgeons. The program was designed to monitor the rehabilitation process, functional evaluation, discharge planning, and follow-

Jae-Young Lim

Department of Rehabilitation Medicine, Seoul National University Bundang Hospital, Seoul National University College of Medicine, Seongnam, Korea

Corresponding Author: Jae-Young Lim, MD

https://orcid.org/0000-0002-9454-0344

Department of Rehabilitation, Seoul National University Bundang

Hospital, Seoul National University College of Medicine, 82 Gumi-ro

173beon-gil, Bundang-gu, Seongnam 13620, Korea

Tel: +82-31-787-7732, Fax: +82-31-787-4056

E-mail: drlim1@snu.ac.kr

Received: September 16, 2018

Accepted: September 18, 2018 up at 3, 6, and 12 months after surgery. Furthermore, the patients and their caregivers were instructed with regards to follow-up evaluation and management after discharge in order to have them visit outpatient clinics without fail. However, there were many patients who did not visit the outpatient clinics until 6 months after surgery due to various reasons. Some patients died or were accommodated in long-term care facilities; contrarily, some others had already become independent in daily life and did not want to continue with further outpatient follow-ups. In addition, some patients were admitted at other institutions. It could be possible that the patients who had fairly good or very bad function failed to participate in follow-up evaluations. Therefore, selection bias should be considered because the patients who did not visited the outpatient clinics were not included in our study.

In our study, 7 variables were used in multiple logistic regression analysis, including nonmodifiable variables like age and sex as well as Berg Balance Scale (BBS) and Mini-Mental State Examination (MMSE) scores; BBS and MMSE scores were significantly different between the good-outcome and poor-outcome groups in the univariate analysis. As to the factors reported in previous studies, we referred to those as factors that had significant effects on the functional outcomes of patients with hip fractures. The variable of geriatric depression scale (GDS) score was not included in the multiple logistic regression analysis because the differences in GDS scores between the 2 groups were not significant.

As already mentioned in our study, prospective observational studies are urgently needed because of the apparent limitations of the retrospective study design. Therefore, we developed a standardized program called the fragility fracture-integrated rehabilitation management after hip fracture (FIRM-HIP). Since 2016, a nationwide study on fragility fractures is being conducted that involves a prospective cohort of older patients with fragility fractures who were chosen by referencing the cohort established by the National Strategic Coordinating Center for Clinical Research. ${ }^{3)}$ In addition, a comparative effectiveness study has been conducted to compare the effectiveness of the FIRM program with those of conventional postoperative rehabilitation and historical control treatments. ${ }^{4}$ It is expected that this trial will acquire more evidence on the effectiveness of Fracture Liaison Services focused on rehabilitation, 
which will help determine if these services are fit for medical insurance and health care delivery system of Korea.

\section{CONFLICTS OF INTEREST DISCLOSURES}

The researcher claims no conflicts of interest.

\section{REFERENCES}

1. Kang JH, Lee G, Kim KE, Lee YK, Lim JY. Determinants of functional outcomes using clinical pathways for rehabilitation after hip fracture surgery. Ann Geriatr Med Res 2018;22:26-32.
2. Kim BR. Letter to the Editor: Determinants of functional outcomes using clinical pathways for rehabilitation after hip fracture surgery. Ann Geriatr Med Res 2018;22:105-6

3. Park YG, Nam KW, Kim SR, Park MS, Kim SJ, Ha YC. Improvement of osteoporosis medication after multimodal intervention in patients with hip fracture: prospective multicenter study. J Bone Metab 2018;25:107-13.

4. Lee SY, Beom J, Kim BR, Lim SK, Lim JY, Fragility Fracture Rehabilitation Study Group. Comparative effectiveness of fragility fracture integrated rehabilitation management for elderly individuals after hip fracture surgery: a study protocol for a multicenter randomized controlled trial. Medicine (Baltimore) 2018;97:e10763. 